\title{
Comparative study of index of refraction: The strongly driven and the degenerate two-level systems
}

\author{
R.-H. Rinkleff ${ }^{1,2}$, L. Spani Molella ${ }^{1,2}$, A. Rocco ${ }^{2}$, A. Wicht ${ }^{2}$, K. Danzmann ${ }^{1,2}$ \\ ${ }^{1}$ Institut für Gravitationsphysik, Leibniz Universität Hannover und \\ ${ }^{2}$ Albert Einstein Institut, Max Plack Institut für Gravitationsphysik, D-30167 Hannover, Callinstr. 38, \\ E-mail: rolf-hermann.rinkleff@aei.mpg.de
}

It is well known that quantum coherence and interference are import mechanisms for designing the optical properties of atoms and molecules. Examples are enhancing the index of refraction, large optical Kerr nonlinearities, and cancellation of absorption. These media provide a variety of new applications, such as ultraslowing of light propagation, superluminal light propagation, ultra-sensitive magnetometer or high-finesse broadband optical cavities.

In this contribution we compare the experimental results of absorption and refractive index of a probe laser measured in a strongly driven two-level atom with the results measured in a degenerate two-level system using pump-probe spectroscopy. We demonstrate the possibility of realization an anomalous dispersive transparent medium using the strongly driven $4 \mathrm{~s}^{2}{ }^{1} \mathrm{~S}_{0}-4 \mathrm{~s} 4 \mathrm{p}{ }^{1} \mathrm{P}_{1} \mathrm{Ca}$ transition. Absorption and phase shift profiles were measured as function of the driving field intensity using a $\mathrm{Ca}$ atomic beam. With increasing driving field intensities absorption and phase shift decrease, but the absorption decreases stronger than the dispersion [1]. We find that the resonant absorption and the anomalous dispersion are reduced with respect to the non-driving case by a factor 490 and 47 , respectively. This means a $\sim 10$-fold enhancement of the amount of dispersion provided per amount of absorption.

Absorption and dispersion spectra were measured in the two closed degenerate two-level transitions in the caesium D2 line, when a probe and a coupling laser simultaneously probed and coupled the hyperfine structure in an atomic beam. The absorption and dispersion spectra of the probe laser were measured in dependence of the coupling laser intensity. At the two-photon resonances, the transition $6 \mathrm{~s}^{2} \mathrm{~S}_{1 / 2}, \mathrm{~F}=3-6 \mathrm{p}{ }^{2} \mathrm{P}_{3 / 2}, \mathrm{~F}=2$ shows enhanced transparency (EIT) and the transition $6 \mathrm{~s}^{2} \mathrm{~S}_{1 / 2}, \mathrm{~F}=4-6 \mathrm{p}^{2} \mathrm{P}_{3 / 2}, \mathrm{~F}=5$ enhanced absorption. The first case results in normal dispersion, while the second one corresponds to anomalous dispersion. At low intensities, when power broadening is not significant, the absolute values of the dispersion in both transitions increase with the coupling-field intensity. The dispersion saturates when the resonance width is determined by power broadening. For higher intensities the dispersion decreases with the intensity. In a figure of merit, the ratio between the refractive-index non-unitarian part (n-1) and the absorption coefficient $(\alpha)$ measured in conditions of electromagnetically induced transparency shows a maximum value, which results for a coupling power of 3.18 $\mathrm{mW} 100000$ times larger than in the absence of the coupling laser field [2].

This work was possible through the financial support of the grant no. SFB407 of the Deutsche Forschungsgemeinschaft.

[1] A. Rocco, Dissertation, Hannover

[2] L. Spani Molella, R. - H. Rinkleff, G. Kühn, K. Danzmann, „Giant Kerr effect in closed degenerate two-level transitions,“ Appl. Phys. B (accepted) 